\title{
Diverse genomoviruses representing twenty-nine species identified associated with plants
}

\author{
Rafaela S. Fontenele ${ }^{1,2} \cdot$ Philippe Roumagnac $^{3,4} \cdot$ Cécile Richet $^{3,4} \cdot$ Simona Kraberger $^{1} \cdot$ Daisy Stainton $^{5}$. \\ Maketalena Aleamotu'a ${ }^{6}$. Denis Filloux ${ }^{3,4}$. Pauline Bernardo ${ }^{3,4,7}$. Gordon W. Harkins ${ }^{8}$. James McCarthy ${ }^{9}$. \\ Lachlan S. Charles ${ }^{10} \cdot$ Natalia S. Lamas $^{11}$ - Emanuel F. M. Abreu ${ }^{11}$ - Rayane A. Abreu ${ }^{11,12}$. Graciete B. Batista ${ }^{11,12}$. \\ Ana L. M. Lacerda ${ }^{11}$. Andrew Salywon ${ }^{13} \cdot$ Martin F. Wojciechowski ${ }^{2} \cdot$ Lucas C. Majure $^{14} \cdot$ Darren P. Martin $^{15}$. \\ Simone G. Ribeiro ${ }^{11,12} \cdot$ Pierre Lefeuvre ${ }^{16} \cdot$ Arvind Varsani $^{1,2,17}$
}

Received: 19 May 2020 / Accepted: 9 August 2020 / Published online: 6 September 2020

(c) Springer-Verlag GmbH Austria, part of Springer Nature 2020

\begin{abstract}
Genomoviruses (family Genomoviridae) are circular single-stranded DNA viruses that have been mainly identified through metagenomics studies in a wide variety of samples from various environments. Here, we describe 98 genomes of genomoviruses found associated with members of 19 plant families from Australia, Brazil, France, South Africa and the USA. These 98 genomoviruses represent 29 species, 26 of which are new, in the genera Gemykolovirus $(\mathrm{n}=37)$, Gemyduguivirus $(\mathrm{n}=$ $9)$, Gemygorvirus $(\mathrm{n}=8)$, Gemykroznavirus $(\mathrm{n}=6)$, Gemycircularvirus $(\mathrm{n}=21)$ and Gemykibivirus $(\mathrm{n}=17)$.
\end{abstract}

The family Genomoviridae was established in 2016 [15] following the identification and characterization of the

Handling Editor: Elvira Fiallo-Olivé.

Electronic supplementary material The online version of this article (https://doi.org/10.1007/s00705-020-04801-5) contains supplementary material, which is available to authorized users.

Arvind Varsani

arvind.varsani@asu.edu

1 The Biodesign Center for Fundamental and Applied Microbiomics and Center for Evolution and Medicine, Arizona State University, Tempe, AZ 85287-5001, USA

2 School of Life sciences, Arizona State University, Tempe, AZ 85287-5001, USA

3 CIRAD, BGPI, 34398 Montpellier, France

4 BGPI, INRAE, CIRAD, Institut Agro, Univ Montpellier, 34398 Montpellier, France

5 Department of Entomology and Plant Pathology, Division of Agriculture, University of Arkansas System, Fayetteville, AR 72701, USA

6 School of Environmental and Life Sciences, The University of Newcastle, Callaghan, NSW 2308, Australia

7 Enza Zaden, Haling 1-E, 1602 DB Enkhuizen, The Netherlands

8 South African MRC Bioinformatics Unit, South African National Bioinformatics Institute, University of the Western Cape, Bellville, South Africa single-stranded DNA (ssDNA) virus Sclerotinia sclerotiorum hypovirulence-associated DNA virus 1 (SsHADV-1) [24] and further isolation of several similar sequences from a wide variety of samples [22] including plants [3, 5, 6, 9, $14,16,18,19]$. SsHADV-1 was found infecting and causing

9 Manaaki Whenua, Landcare Research, Lincoln 7640, New Zealand

10 Department of Botany and Plant Sciences, University of California, Riverside, CA 92507, USA

11 Embrapa Recursos Genéticos e Biotecnologia, Brasília, DF, Brazil

12 PPG Ciências Naturais e Biotecnologia, Universidade Federal de Campina Grande, Cuité, PB, Brazil

13 Desert Botanical Garden, Phoenix, AZ 85008, USA

14 Florida Museum of Natural History, University of Florida, Gainesville, FL 32611, USA

15 Computational Biology Division, Department of Integrative Biomedical Sciences, Institute of Infectious Diseases and Molecular Medicine, University of Cape Town, Observatory 7925, South Africa

16 CIRAD, UMR PVBMT, St. Pierre, 97410 La Réunion, France

17 Structural Biology Research Unit, Department of Clinical Laboratory Sciences, University of Cape Town, Observatory, 7925 Cape Town, South Africa 
hypovirulence in the plant fungal pathogen Sclerotinia sclerotiorum and currently is the only genomovirus that has been experimentally associated with a host; the other isolated genomoviruses have no further host confirmation. The family Genomoviridae has been divided into nine genera based on the phylogeny of their replication-associated protein (Rep) amino acid sequence. These genera are Gemycircularvirus, Gemyduguivirus, Gemygorvirus, Gemykibivirus, Gemykolovirus, Gemykrogvirus, Gemykroznavirus, Gemytondvirus and Gemyvongvirus [22].

Genomovirus genomes are circular, between $\sim 2.0$ and $2.3 \mathrm{~kb}$ in length, and have at least three large open reading frames (ORFs). These ORFs encode a Rep on the complementary-sense genome strand that is likely expressed from a spliced transcript, a putative RepA protein on the complementary strand, and a putative capsid protein (CP) on the virion strand. The Reps of genomoviruses are evolutionarily most closely related to those of plant-infecting viruses of the family Geminiviridae [22]. Unlike geminiviruses, however, genomoviruses have no recognizable movement protein genes, and their capsid proteins (CPs) have no easily detectable homology to those of any other classified viruses [25].

While the vast majority of known plant viruses have been discovered in cultivated plants, less is known about the diversity and pathogenicity of viruses infecting noncultivated plants (i.e., wild plants, including weeds) [23]. Additional information on the diversity of viruses associated with non-cultivated plants will provide critical new information on the ecology and evolution of plant viruses beyond the few cultivars tested to date.

In this study, we report the identification of 98 genomovirus genomes associated with 88 plant samples representing 19 plant families collected between 2008 and 2018 in Australia, Brazil, France, South Africa and the USA (Table 1). In the Western Cape Region of South Africa, plants were sampled randomly between 2010 and 2012 regardless of infection symptoms within a $4.5 \mathrm{~km} \times 4.5 \mathrm{~km}$ plot [2]. In Northeast Brazil, both viral symptomatic and asymptomatic plants were collected between 2008 and 2015. In Australia, France and the USA, plants were opportunistically sampled regardless of symptoms. The genera and species of sampled plants were identified by expert botanists and from the sequences obtained by high-throughput sequencing. The virion-associated nucleic acids (VANA) method was used to process the samples collected in South Africa as described previously $[2,10]$. Total DNA from the Australian, Brazilian, French and US samples was extracted using the Dellaporta method [7] and enriched for small circular DNA molecules by rolling-circle amplification (RCA) using TempliPhi (GE Healthcare, USA). RCA products were pooled according to sampling location and the taxonomic plant family from which the samples were obtained, and these pooled samples were then sequenced at Macrogen Inc. (South Korea) using the
Illumina HiSeq 2500 platform with $2 \times 150$ bp paired-end sequencing. The paired-end reads were assembled de novo using ABySS 2.0 [12], and contigs > $750 \mathrm{nt}$ were compared to the viral GenBank sequence database using BLASTx [1]. Abutting primers were designed for contigs displaying detectable homology to known genomoviruses, and these were used to recover the full genomovirus genomes using PCR employing Kapa Hotstart HiFi polymerase (Kapa Biosystems, USA) and RCA products from the different plant samples as templates with the thermal cycler conditions recommended by Kapa Biosystems and an annealing temperature of $60^{\circ} \mathrm{C}$. Amplicons of $\sim 2 \mathrm{~kb}$ were resolved on a $0.7 \%$ agarose gel, excised, and eluted using a MEGAquick-spin ${ }^{\mathrm{TM}}$ Total Fragment DNA Purification Kit (iNtRON Biotechnology, South Korea). The resulting DNA fragments were then cloned in pJET 1.2 (Thermo Fisher Scientific, USA), and the corresponding recombinant plasmid was sequenced by the Sanger method at Macrogen Inc. (South Korea). Finally, Geneious v11.1.4 [13] was used to assemble the sequence and annotate the genomovirus genome sequences.

All 416 full-length genomovirus genome sequences available in GenBank on the $1^{\text {st }}$ of February 2020 were analyzed together with the 98 genome sequences determined in this study. Genome-wide pairwise nucleotide sequence identities, and pairwise amino acid sequence identities of Rep and CP were determined using SDT v1.2 [20]. The 98 genomovirus genomes represent 29 species (26 of which are new) based on the currently accepted genomovirus pairwise nucleotide sequence identity species demarcation threshold of 78\% [22] (Supplementary Table 1 and Supplementary Table 2). The genomoviruses recovered in this study have been tentatively named" plant-associated genomovirus (PaGmV) 1 - 29". These new PaGmV sequences range in size from $~ 2.1-2$ to $4 \mathrm{~kb}$ and, as with other known genomoviruses, have a conserved nonanucleotide within their intergenic regions that is the likely origin of virion-strand replication (Table 1). Also, as with other known genomovirus sequences, the new sequences also encode a probable $\mathrm{CP}$ on their presumed virion strand and both a putatively spliced Rep and a RepA on their presumed complementary strands.

A maximum-likelihood phylogenetic tree was inferred from the aligned Rep sequences using PHYML [11] with the $\mathrm{rtRev}+\mathrm{G}$ amino acid substitution model (determined to be the best-fitting model by ProtTest [4]). Branches with approximate likelihood ratio test (aLRT) support $<0.8$ were collapsed using TreeGraph 2 [21]. Geminivirus Rep sequences were used to root the phylogenetic tree. The resulting phylogenetic tree shows that the $\mathrm{PaGmVs}$ could be classified as belonging to six out of the nine recognized genomovirus genera. PaGmV 7-10 $(n=37)$ were tentatively assigned to the genus Gemykolovirus, PaGmV 4-5 $(\mathrm{n}=9)$ to the genus Gemyduguivirus, $\mathrm{PaGmV} 1$ and $26(\mathrm{n}=8)$ to the genus Gemygorvirus, and PaGmV 23-24 $(\mathrm{n}=6)$ to the 


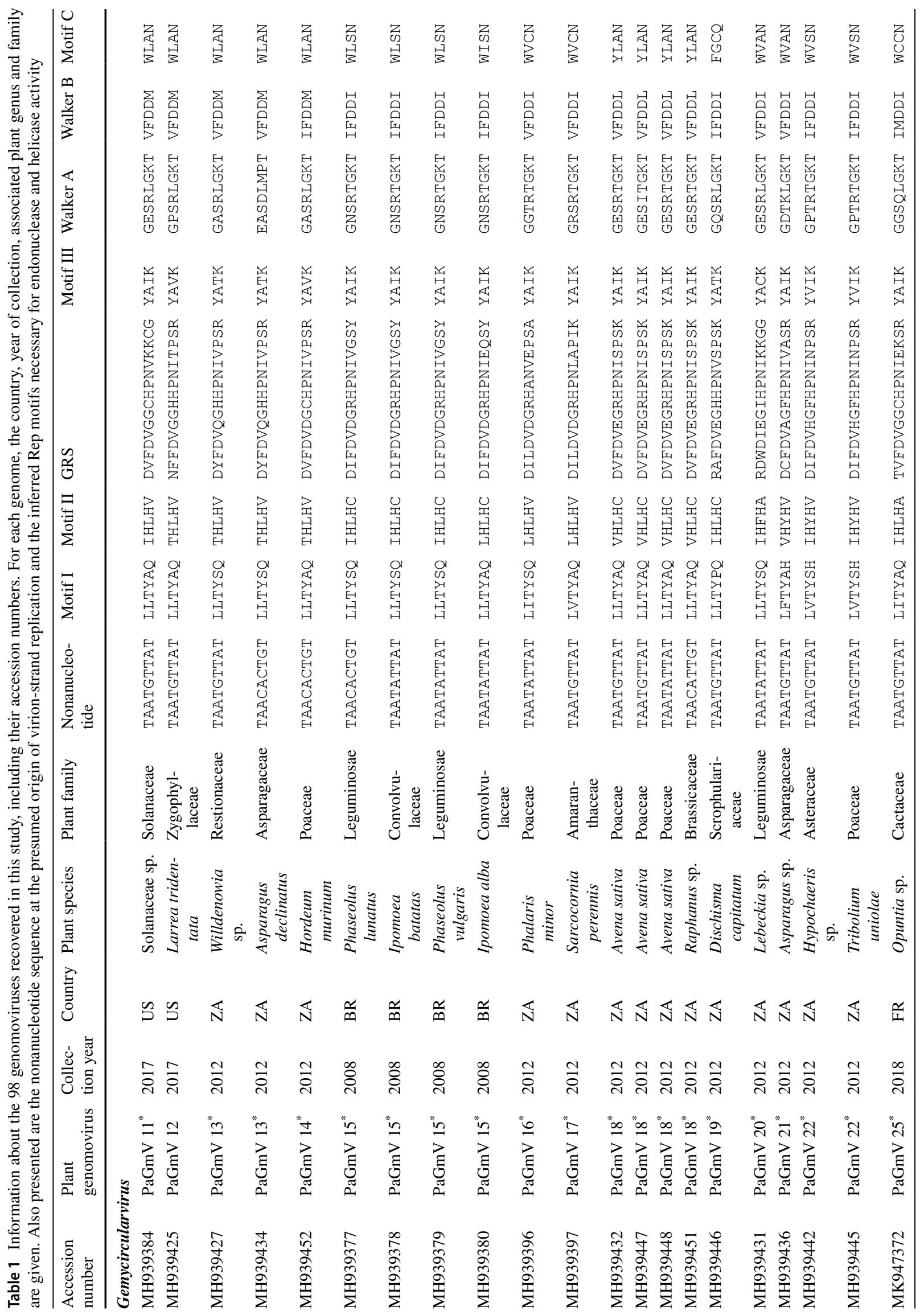




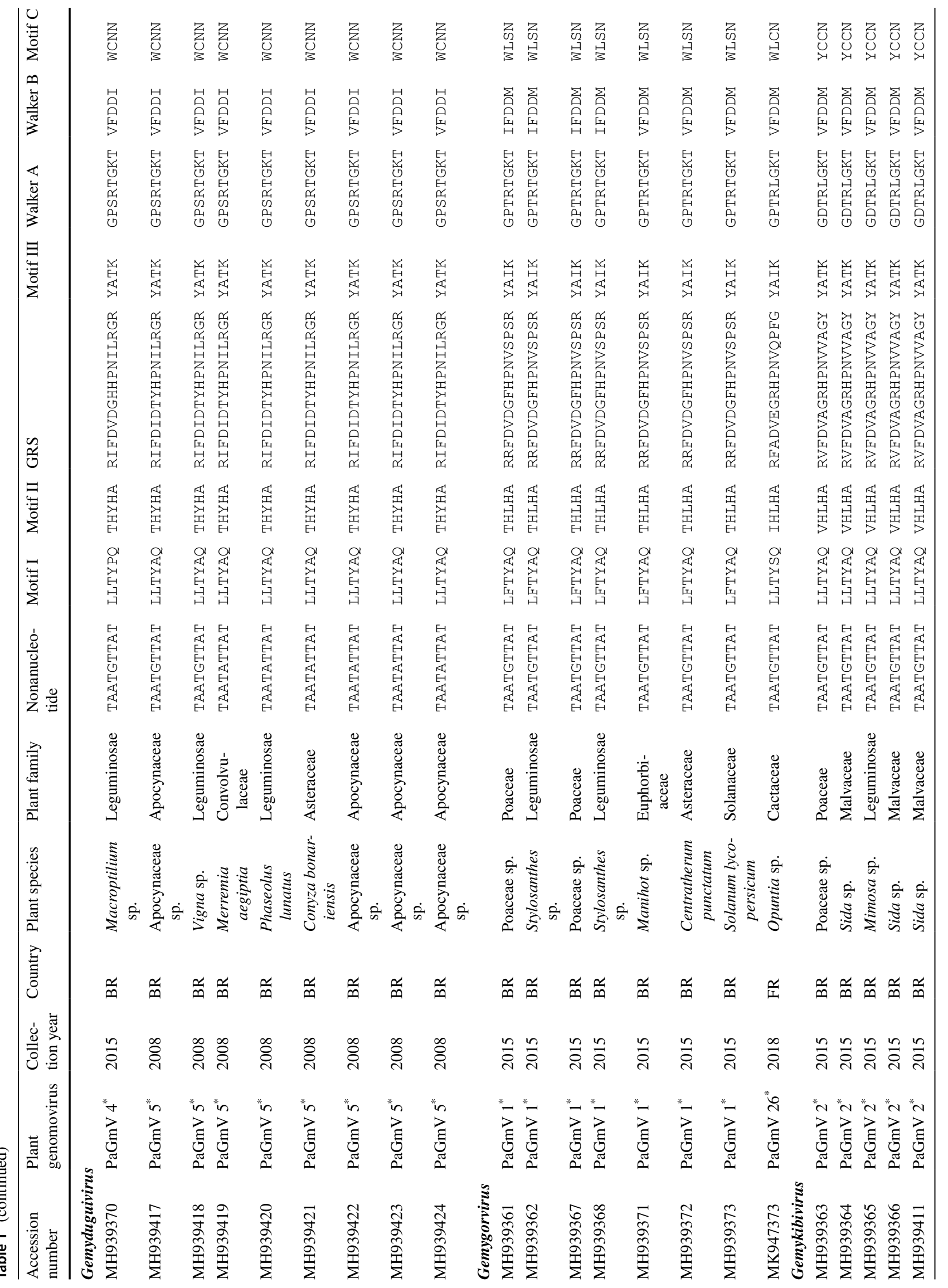




\begin{tabular}{|c|c|c|c|c|c|c|c|c|c|c|c|c|c|c|c|}
\hline$\sum^{\circ}$ & $\underbrace{Z}_{\substack{u \\
\hdashline}}$ & 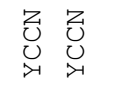 & 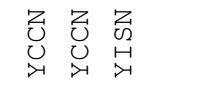 & 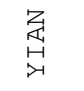 & 蛋 & 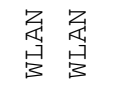 & $\begin{array}{l}2 \\
\text { 足 } \\
1 \\
3\end{array}$ & $\begin{array}{l}2 \\
\text { 足 } \\
1 \\
3\end{array}$ & 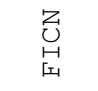 & $\begin{array}{l}Z \\
\text { U } \\
H \\
H\end{array}$ & 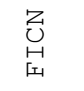 & 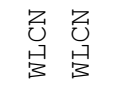 & $\begin{array}{l}Z_{0} \\
\text { 夏 }\end{array}$ & $\begin{array}{l}z \\
0 \\
\text { 恶 }\end{array}$ & $\begin{array}{l}Z \\
\text { 己 } \\
\text { 兵 }\end{array}$ \\
\hline$\frac{5}{\pi}$ & $\begin{array}{l}\sum_{1} \\
\text { 空 } \\
\text { 足 }\end{array}$ & 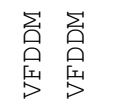 & 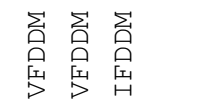 & 窝 & $\begin{array}{l}\sum_{1} \\
\text { 号 } \\
\text { 窝 }\end{array}$ & 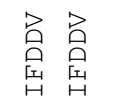 & 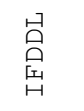 & 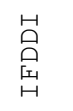 & 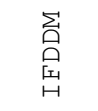 & 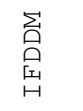 & 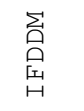 & 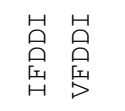 & 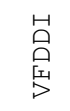 & $\begin{array}{l}\stackrel{H}{\circ} \\
\text { 窝 } \\
\text { H }\end{array}$ & 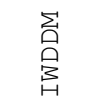 \\
\hline & 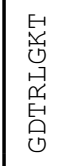 & 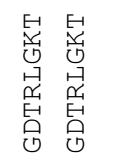 & 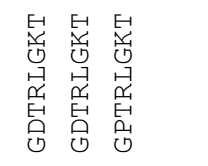 & 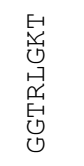 & 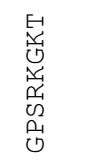 & 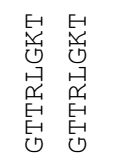 & 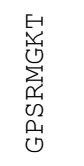 & 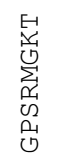 & 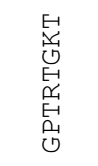 & 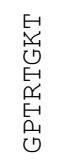 & 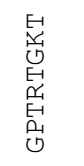 & 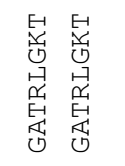 & 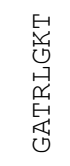 & 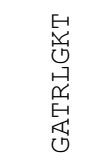 & 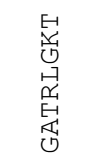 \\
\hline & 崫 & 总祭 & 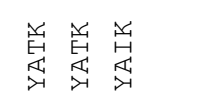 & 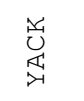 & 离 & 乩乩 & 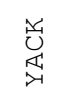 & 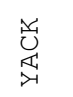 & 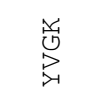 & $\begin{array}{l}\text { 苟 } \\
\text { 究 }\end{array}$ & $\begin{array}{l}\infty \\
0 \\
0 \\
\sum_{\lambda=1}^{\infty}\end{array}$ & 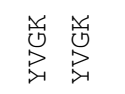 & $\begin{array}{l}\text { 苟 } \\
\text { 究 }\end{array}$ & $\begin{array}{l}\text { 苟 } \\
\text { 究 }\end{array}$ & 范 \\
\hline 난 & 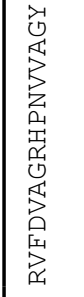 & 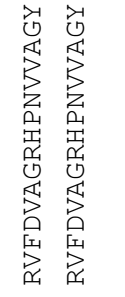 & 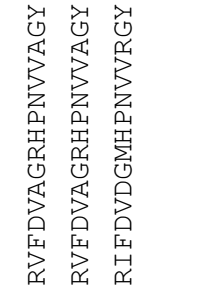 & 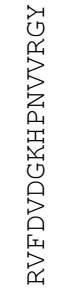 & 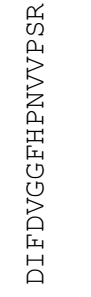 & 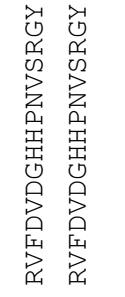 & 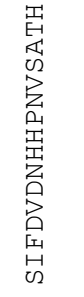 & 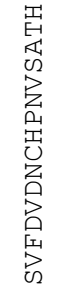 & 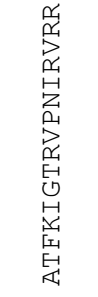 & 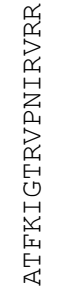 & 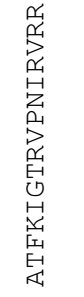 & 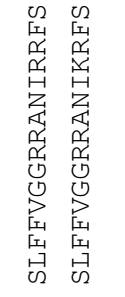 & 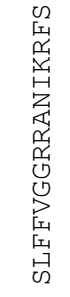 & 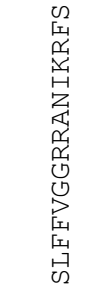 & 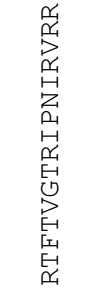 \\
\hline & $\begin{array}{l}\text { 頱 } \\
\text { 窝 }\end{array}$ & 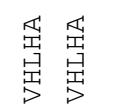 & 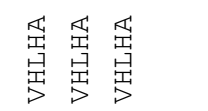 & 蛓 & 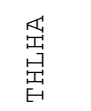 & 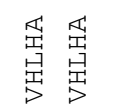 & $\begin{array}{l}\text { 虫 } \\
\text { 蚰 }\end{array}$ & 強 & $\begin{array}{l}\text { 萺 } \\
\text { 虫 }\end{array}$ & 秮 & $\begin{array}{l}\text { 蜔 } \\
\text { 蜔 }\end{array}$ & 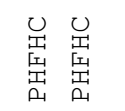 & 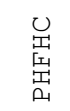 & $\begin{array}{l}\text { 足 } \\
\text { 虫 } \\
\text { 虫 }\end{array}$ & $\begin{array}{l}\text { 蚛 } \\
\text { 密 } \\
\text { 虫 }\end{array}$ \\
\hline & 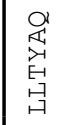 & 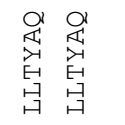 & 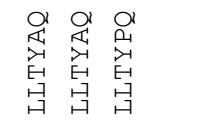 & 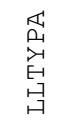 & 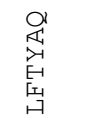 & 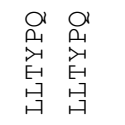 & 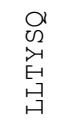 & $\begin{array}{l}\text { a } \\
\text { on } \\
\text { 文 } \\
\text { 珀 }\end{array}$ & 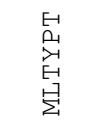 & 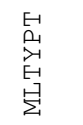 & 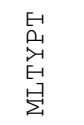 & 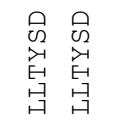 & 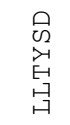 & 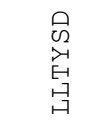 & 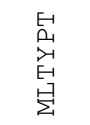 \\
\hline 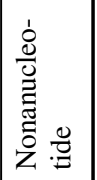 & 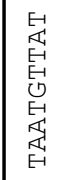 & 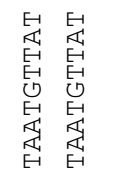 & 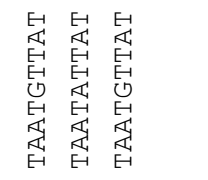 & 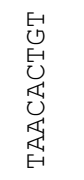 & 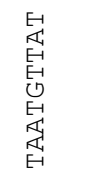 & 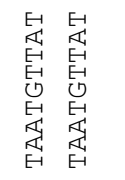 & 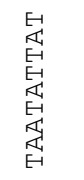 & 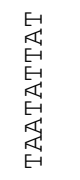 & 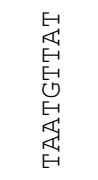 & 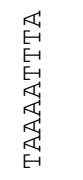 & 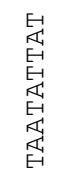 & 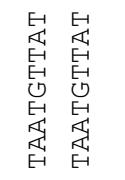 & 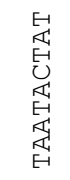 & 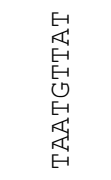 & 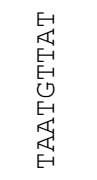 \\
\hline 高 & 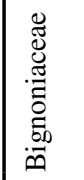 & 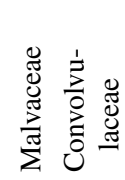 & 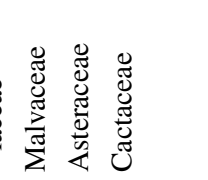 & 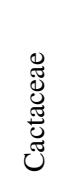 & 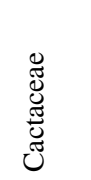 & 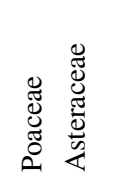 & 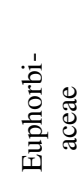 & 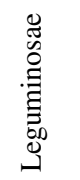 & 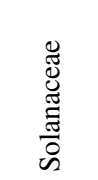 & 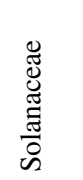 & 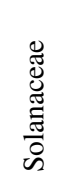 & 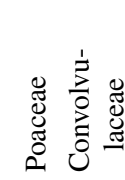 & 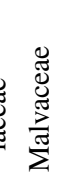 & 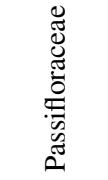 & 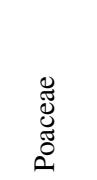 \\
\hline 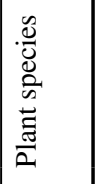 & 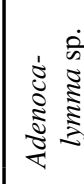 & 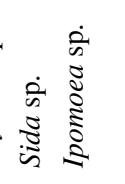 & 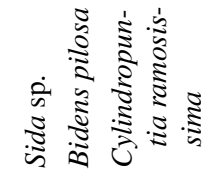 & 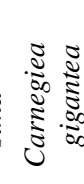 & 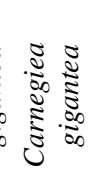 & 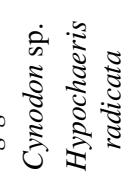 & $\begin{array}{l}\dot{2} \\
\frac{2}{5} \\
\tilde{\Xi}\end{array}$ & $\frac{\sqrt{-1}}{-3}$ & 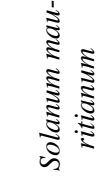 & 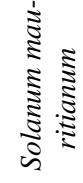 & 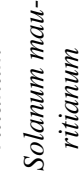 & 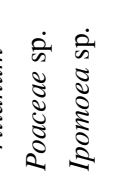 & 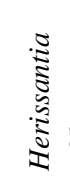 & 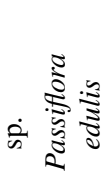 & 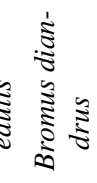 \\
\hline U & $\frac{\alpha}{\infty}$ & $\stackrel{\frac{\alpha}{m}}{a}$ & 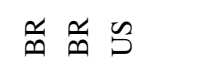 & $\tilde{s}$ & $\tilde{s}$ & $\mathbb{N} \mathbb{N}$ & $\stackrel{n}{\infty}$ & $\overleftrightarrow{N}$ & 导 & 2 & 2 & $\frac{n}{m} \frac{d}{m}$ & $\frac{\alpha}{\infty}$ & $\frac{\alpha}{\infty}$ & $\mathbb{N}$ \\
\hline 㽕 & $\stackrel{n}{\tilde{N}}$ & 号 & 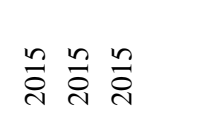 & $\hat{\text { ิㅗ }}$ & $\overrightarrow{\bar{i}}$ & 롤 & $\stackrel{n}{\grave{N}}$ & ڤั & $\frac{n}{i}$ & 号 & $\stackrel{n}{\stackrel{n}{n}}$ & $\stackrel{n}{\stackrel{n}{*}} \stackrel{n}{\bar{N}}$ & $\stackrel{n}{\grave{n}}$ & $\stackrel{n}{\grave{2}}$ & $\stackrel{\sim}{\tilde{N}}$ \\
\hline 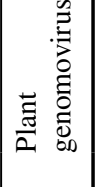 & 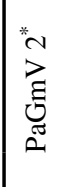 & 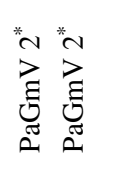 & 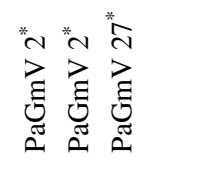 & 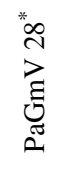 & 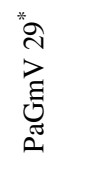 & 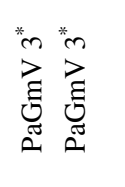 & 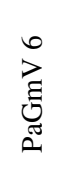 & 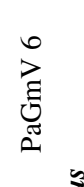 & 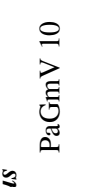 & 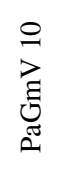 & $\begin{array}{l}0 \\
2 \\
\text { ह } \\
\text { ह } \\
0\end{array}$ & 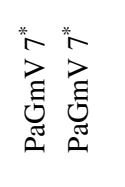 & 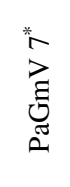 & 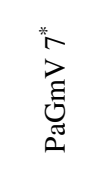 & 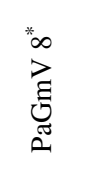 \\
\hline 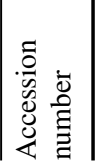 & 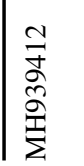 & 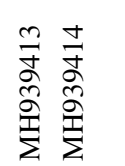 & 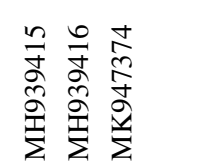 & \begin{tabular}{l}
$n$ \\
$\hat{2}$ \\
\multirow{2}{*}{} \\
$\stackrel{2}{2}$
\end{tabular} & 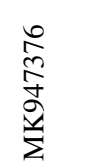 & 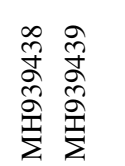 & 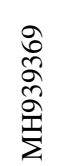 & 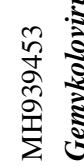 & 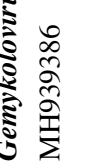 & 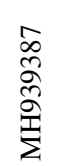 & 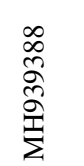 & 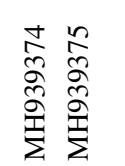 & 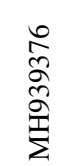 & 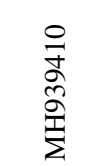 & $\begin{array}{l}\bar{\infty} \\
\text { } \\
\stackrel{\hat{\sigma}}{\Sigma} \\
\stackrel{\Sigma}{\Sigma}\end{array}$ \\
\hline
\end{tabular}




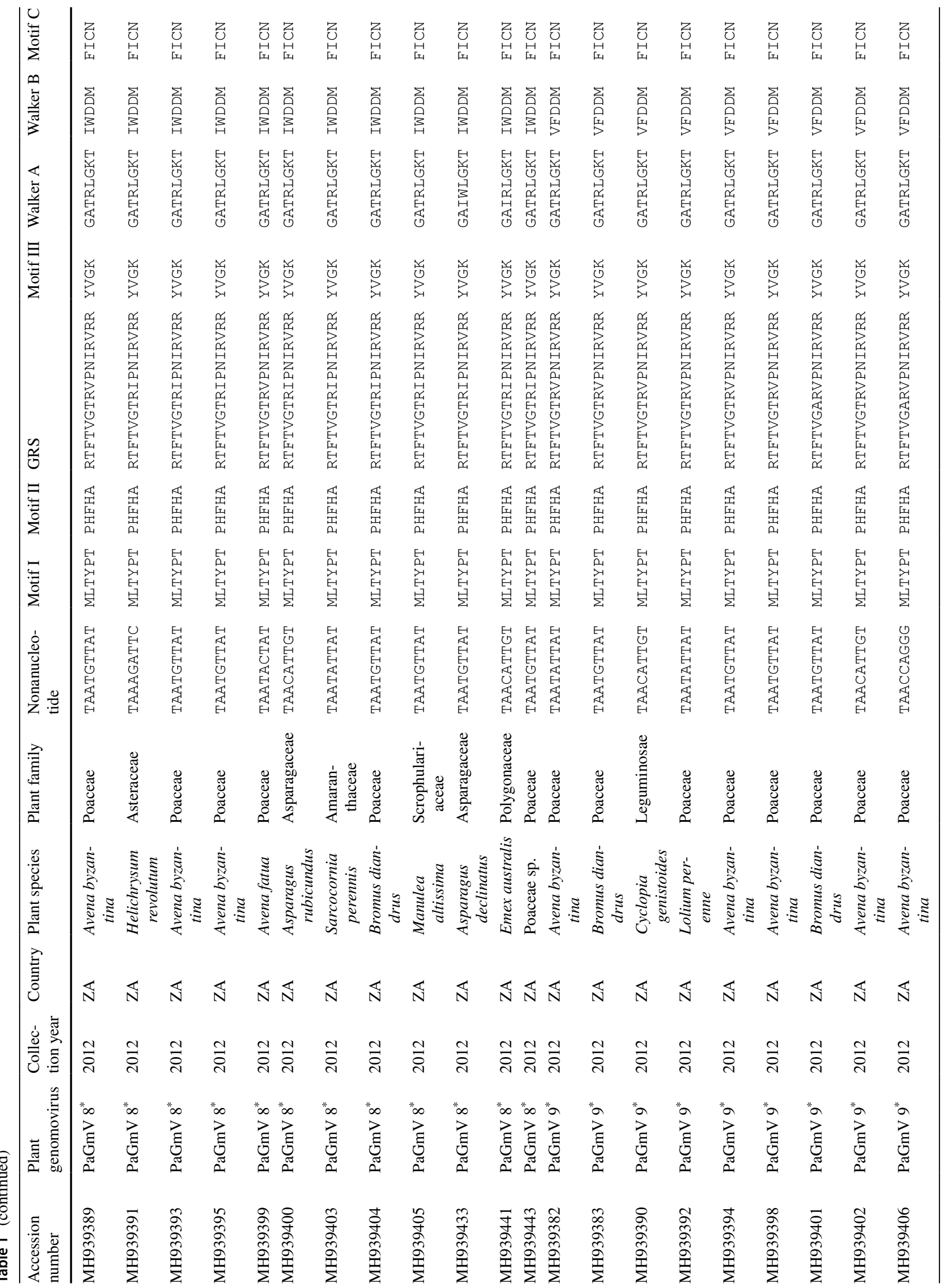




\begin{tabular}{|c|c|c|c|c|c|c|}
\hline$\sum_{\bar{z}}^{0}$ & 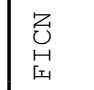 & $\begin{array}{l}\text { 总 } \\
\text { 㟉 }\end{array}$ & $\begin{array}{l}Z_{0} \\
\text { 早 } \\
\text { 出 }\end{array}$ & 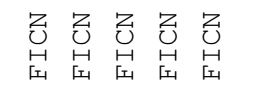 & $\begin{array}{ll}2 & 2 \\
0 & 0 \\
0 & 0 \\
3 & 3\end{array}$ & 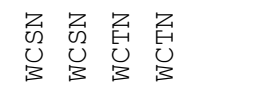 \\
\hline & $\begin{array}{l}\sum_{1} \\
\text { 空 } \\
\text { 品 }\end{array}$ & $\begin{array}{l}\sum_{1} \\
\text { 空 } \\
\text { 站 }\end{array}$ & 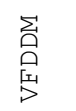 & 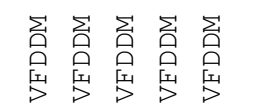 & 拿鬲 & 峝荟峝峝 \\
\hline & 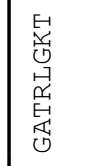 & 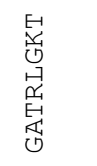 & 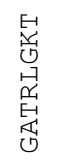 & 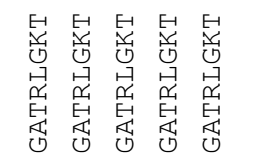 & 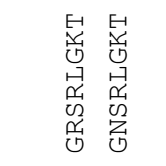 & 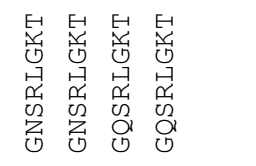 \\
\hline & 盛 & 㭊 & 总 & 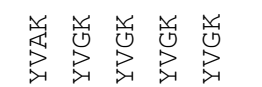 & 总总 & 岛总总总 \\
\hline & 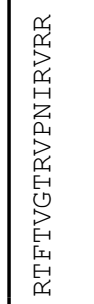 & 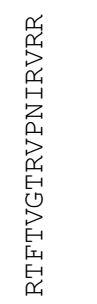 & 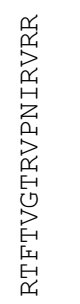 & 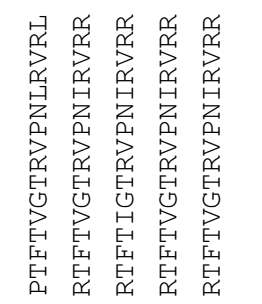 & 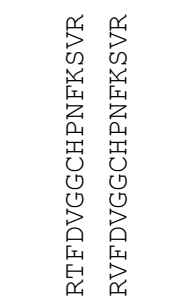 & 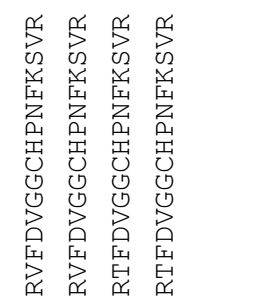 \\
\hline & $\begin{array}{l}\text { 虫 } \\
\text { 虫 } \\
\text { 焉 }\end{array}$ & 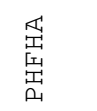 & 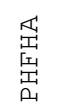 & 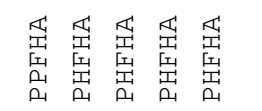 & $\begin{array}{l}\text { 虹虹 } \\
\text { 虫 } \\
\text { 嵒 }\end{array}$ & 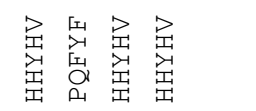 \\
\hline & 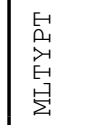 & $\begin{array}{l}\text { 鼠 } \\
\text { 臽 } \\
\text { 帘 }\end{array}$ & 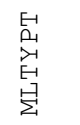 & 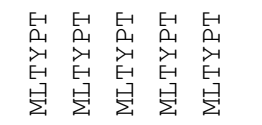 & 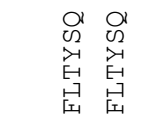 & 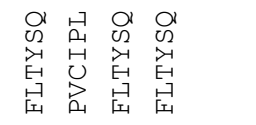 \\
\hline 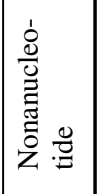 & 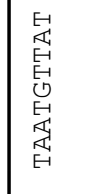 & 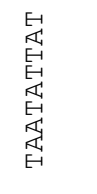 & 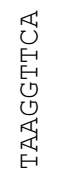 & 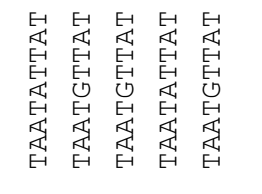 & 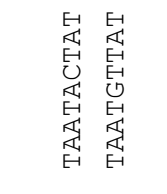 & 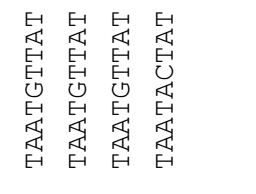 \\
\hline (1) & 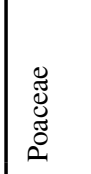 & 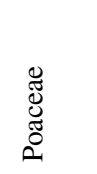 & 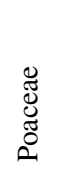 & 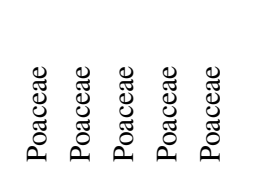 & 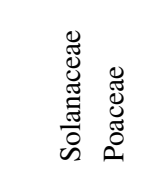 & 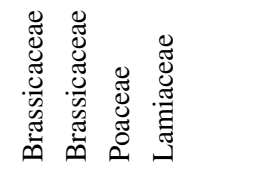 \\
\hline 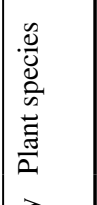 & 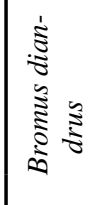 & 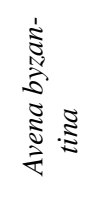 & 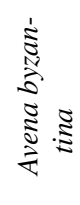 & 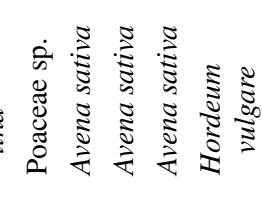 & 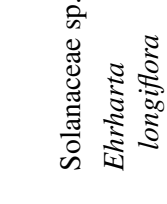 & 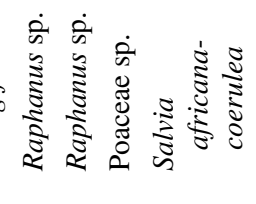 \\
\hline & $\mathbb{N}$ & $\overleftrightarrow{N}$ & $\overleftrightarrow{N}$ & $\mathbb{N} \overleftrightarrow{N} \mathbb{N} \mathbb{N}$ & $\tilde{\rho} \mathbb{N}$ & $\overleftrightarrow{N} \mathbb{N} \mathbb{N}$ \\
\hline 递 & $\stackrel{\text { ㄱ }}{\stackrel{2}{1}}$ & 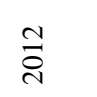 & $\stackrel{\text { กี }}{\stackrel{4}{ }}$ & 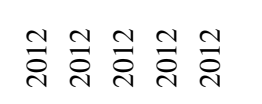 & 혹 & 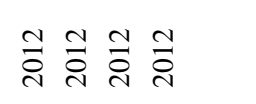 \\
\hline 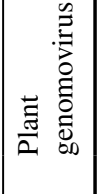 & 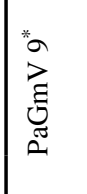 & 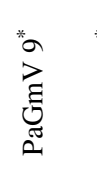 & 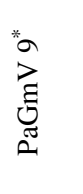 & 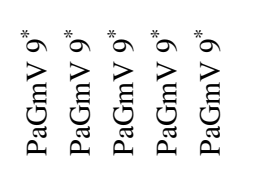 & 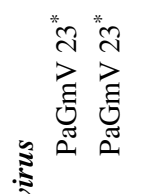 & 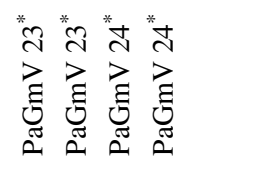 \\
\hline 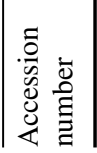 & 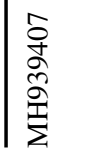 & $\begin{array}{l}\infty \\
\stackrel{+}{\alpha} \\
\text { } \\
\stackrel{1}{\Sigma}\end{array}$ & $\begin{array}{l}\text { के } \\
\text { ळे } \\
\text { 产 }\end{array}$ & 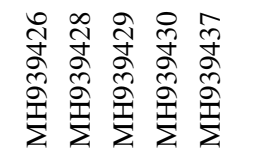 & 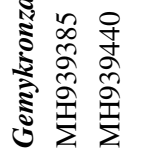 & 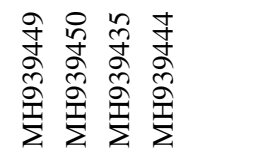 \\
\hline
\end{tabular}




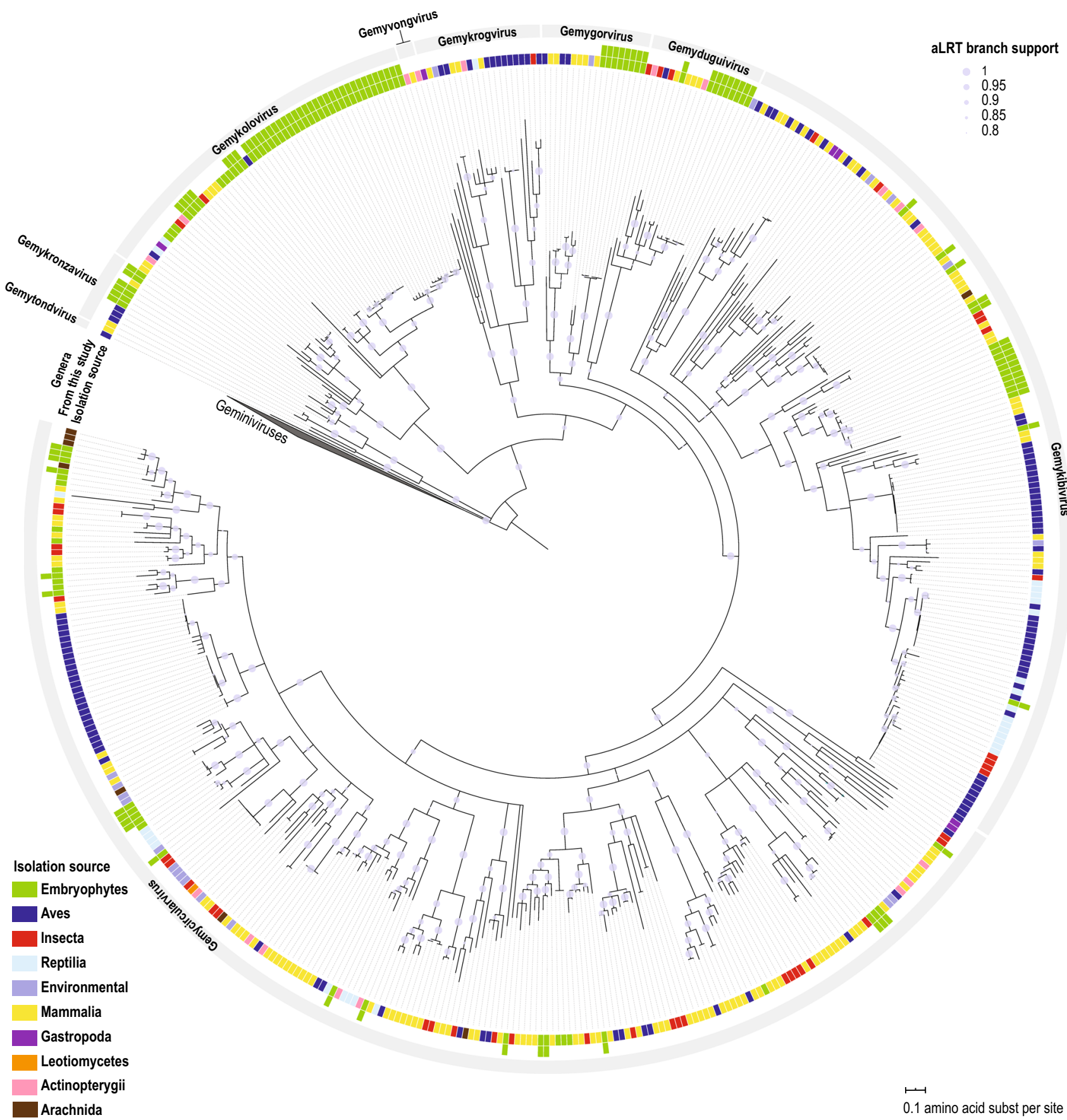

Fig. 1 Maximum-likelihood phylogenetic tree of the Rep amino acid sequences encoded by the genomoviruses from this study in relation to those of other genomoviruses available in the GenBank database (downloaded 1 Feb 2020). The tree was rooted with Rep amino acid sequences from the family Geminiviridae. Branches with $<0.8$ aLRT

genus Gemykroznavirus (Fig. 1). The other PaGmVs were tentatively assigned to two of the larger genomovirus genera with PaGmV 11-22, PaGmV $25(\mathrm{n}=21)$ to the genus Gemycircularvirus, and PaGmV 2-3 and 27-29 $(\mathrm{n}=17)$ to support have been collapsed. The tree is further demarcated by the source from which sequences were obtained: Actinopterygii $(\mathrm{n}=18)$, Arachnida $(n=13)$, Aves $(n=113)$, Embryophyte $(n=116)$; environmental $(n=20)$, Gastropoda $(n=6)$, Insecta $(n=44)$, Leotiomycetes $(n=1)$, Mammalia $(n=155)$, and Reptilia $(n=28)$

the genus Gemykibivirus. The phylogenetic analysis showed that there is no clear correlation between the genomovirus genera and the sample type from which the sequences were obtained (Fig. 1 and Fig. 2B). 
A

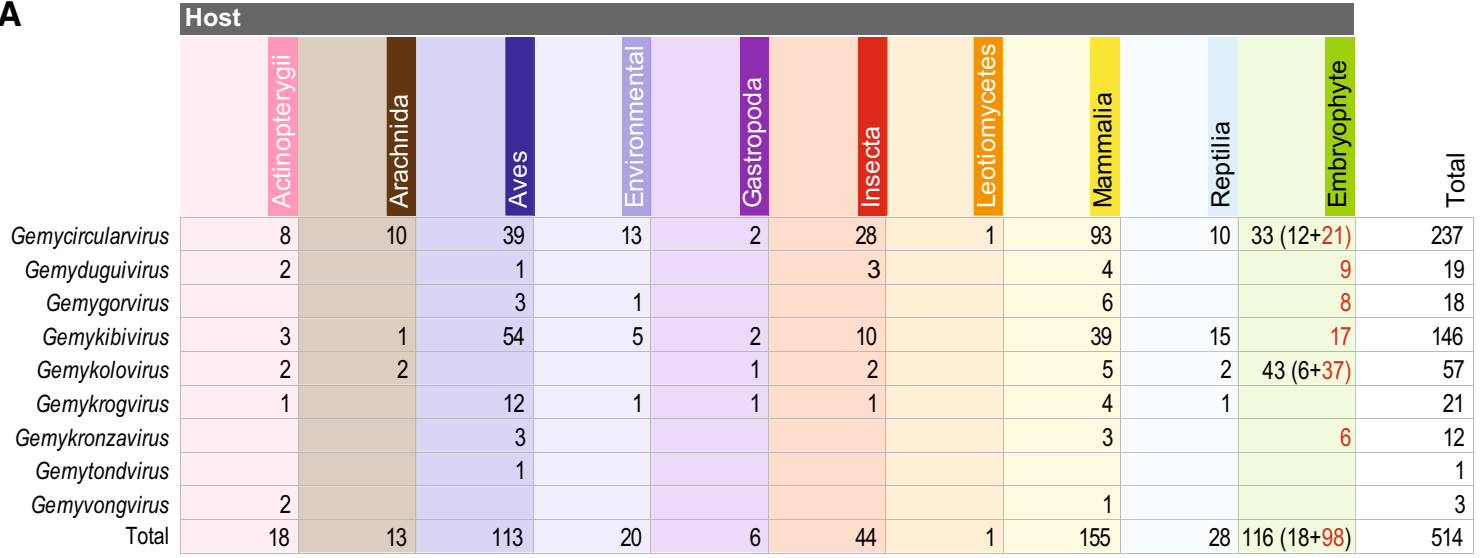

B

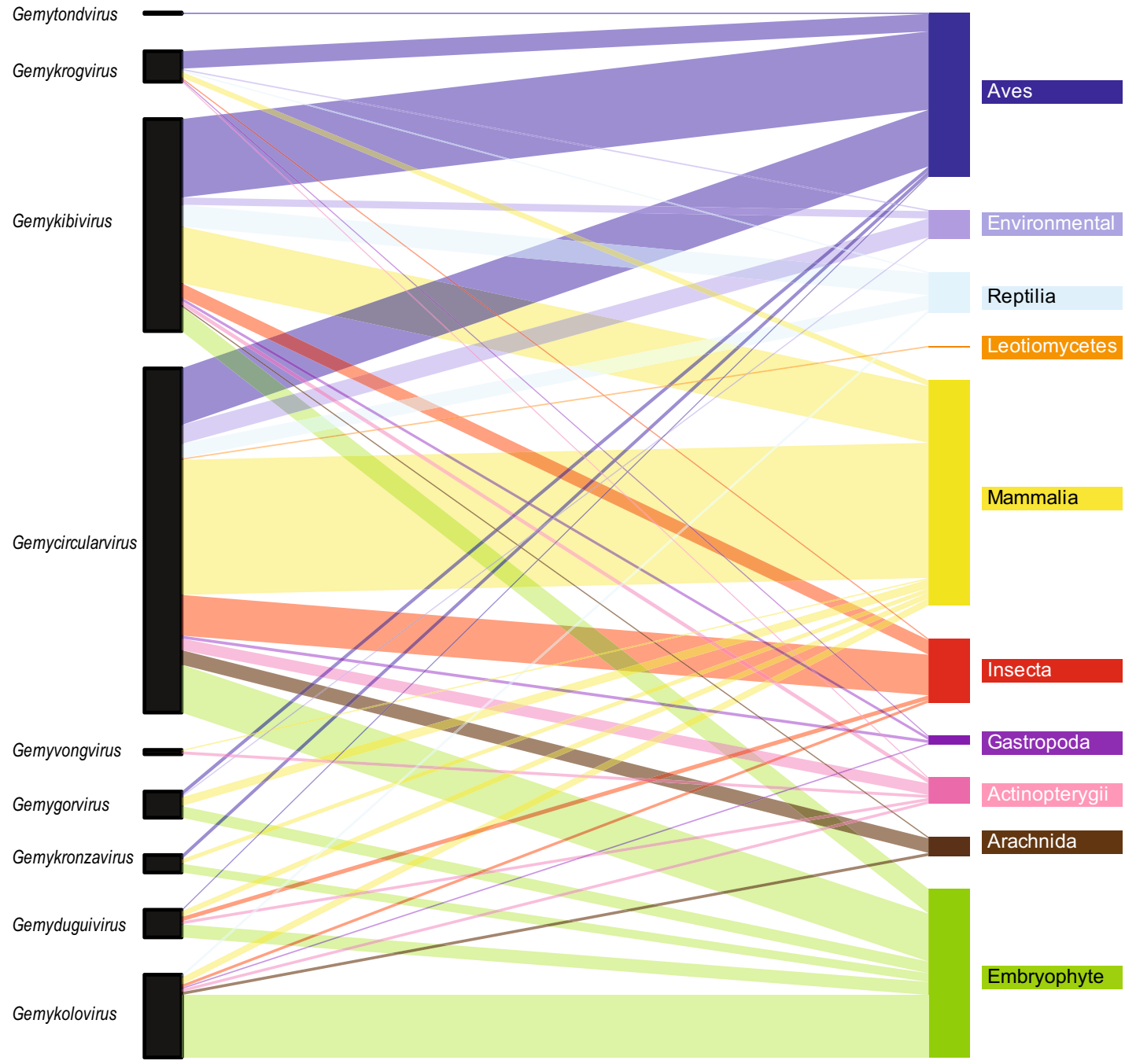

Fig. 2 A. Summary table of the number of viruses identified in this study (highlighted in red font) in relation to those from other sample sources and their genus assignments. B. Bipartitite plot of the host/virus species associations inferred using R with the bipartite graph package [8]

The inferred Rep proteins encoded by the PaGmVs all contain the conserved motifs necessary for endonuclease and helicase activity (Table 1) that are present in most other evolutionarily related Rep proteins. The genome sequences of the $\mathrm{PaGmV}$ s share $>63.3 \%$ pairwise identity with other genomoviruses. The Rep amino acid sequence 
of the PaGmVs share $>63.7 \%$ identity with those of other genomoviruses, while those of their CPs share $>37.9 \%$ identity (Supplementary Table 1).

This study further highlights the broad diversity of genomoviruses that are associated with plants. Before this study, no viruses in the genera Gemykroznavirus, Gemygorvirus, Gemyduguivirus or Gemykibivirus had been found associated with plants. Our research has identified six species of Gemykroznavirus, eight species of Gemygorvirus, nine species of Gemyduguivirus, and 17 species of Gemykibivirus associated with plants (Fig. 2). Additionally, the 37 gemykoloviruses identified here demonstrate that plant-associated viruses are the predominant members of this genus (Fig. 2). Although there are no confirmed hosts for any of the genomoviruses discovered here, it is likely that they infect fungi that are associated with the plants from which they were obtained, but it is also possible that they infect both fungi and their plants hosts. It is probable that additional diverse plant-associated genomoviruses will be identified, especially given the close association between plants and fungi. Furthermore, it is also likely that multipartite genomo-like viruses will be identified that are similar to the tripartite ssDNA virus recently reported infecting Fusarium graminearum, whose Rep shares 24-33\% amino acid sequence identity with genomovirus Reps [17].

Acknowledgements This research was partially funded by grants from Embrapa, CNPq and FAPDF (Brazil). We thank CNPq for the fellowships to NSL and ALML, and to CAPES for the scholarships to RAA and GBB. This research was partially funded by a Student and Early Career Grant awarded to RSF by the Next Generation Sonoran Desert Researchers (N-Gen).

\section{Compliance with ethical standards}

Conflict of interest The authors declare there are no conflicts of interest.

Research involving human participants and/or animals The research did not involve human participants or animals.

\section{References}

1. Altschul SF, Gish W, Miller W, Myers EW, Lipman DJ (1990) Basic local alignment search tool. J Mol Biol 215:403-410

2. Bernardo P, Charles-Dominique T, Barakat M, Ortet P, Fernandez E, Filloux D, Hartnady P, Rebelo TA, Cousins SR, Mesleard F, Cohez D, Yavercovski N, Varsani A, Harkins GW, Peterschmitt M, Malmstrom CM, Martin DP, Roumagnac P (2018) Geometagenomics illuminates the impact of agriculture on the distribution and prevalence of plant viruses at the ecosystem scale. ISME J $12: 173-184$

3. Chabi-Jesus C, Najar A, Fontenele RS, Kumari SG, Ramos-Gonzalez PL, Freitas-Astua J, Kraberger S, Varsani A (2020) Viruses representing two new genomovirus species identified in citrus from Tunisia. Arch Virol:1-5
4. Darriba D, Taboada GL, Doallo R, Posada D (2011) ProtTest 3: fast selection of best-fit models of protein evolution. Bioinformatics 27:1164-1165

5. Dayaram A, Opong A, Jaschke A, Hadfield J, Baschiera M, Dobson RC, Offei SK, Shepherd DN, Martin DP, Varsani A (2012) Molecular characterisation of a novel cassava associated circular ssDNA virus. Virus Res 166:130-135

6. de Rezende RR, Mar TB, Paez LMC, Silva Xavier AD, Xavier CAD, Navas-Castillo J, Zerbini FM, Alfenas-Zerbini P (2018) Complete genome sequences of two gemycircularviruses associated with non-cultivated plants in Brazil. Arch Virol 163:3163-3166

7. Dellaporta SL, Wood J, Hicks JB (1983) A plant DNA minipreparation: version II. Plant Mol Biol Rep 1:19-21

8. Dormann CF, Fründ J, Blüthgen N, Gruber B (2009) Indices, graphs and null models: analyzing bipartite ecological networks. Open Ecol J 2

9. Du Z, Tang Y, Zhang S, She X, Lan G, Varsani A, He Z (2014) Identification and molecular characterization of a singlestranded circular DNA virus with similarities to Sclerotinia sclerotiorum hypovirulence-associated DNA virus 1. Arch Virol 159:1527-1531

10. Filloux D, Dallot S, Delaunay A, Galzi S, Jacquot E, Roumagnac P (2015) Metagenomics approaches based on virion-associated nucleic acids (VANA): an innovative tool for assessing without a priori viral diversity of plants. Methods Mol Biol 1302:249-257

11. Guindon S, Dufayard JF, Lefort V, Anisimova M, Hordijk W, Gascuel O (2010) New algorithms and methods to estimate maximum-likelihood phylogenies: assessing the performance of PhyML 3.0. Syst Biol 59:307-321

12. Jackman SD, Vandervalk BP, Mohamadi H, Chu J, Yeo S, Hammond SA, Jahesh G, Khan H, Coombe L, Warren RL, Birol I (2017) ABySS 2.0: resource-efficient assembly of large genomes using a Bloom filter. Genome Res 27:768-777

13. Kearse M, Moir R, Wilson A, Stones-Havas S, Cheung M, Sturrock S, Buxton S, Cooper A, Markowitz S, Duran C, Thierer T, Ashton B, Meintjes P, Drummond A (2012) Geneious Basic: an integrated and extendable desktop software platform for the organization and analysis of sequence data. Bioinformatics 28:1647-1649

14. Kraberger S, Farkas K, Bernardo P, Booker C, Arguello-Astorga GR, Mesleard F, Martin DP, Roumagnac P, Varsani A (2015) Identification of novel Bromus- and Trifolium-associated circular DNA viruses. Arch Virol 160:1303-1311

15. Krupovic M, Ghabrial SA, Jiang D, Varsani A (2016) Genomoviridae: a new family of widespread single-stranded DNA viruses. Arch Virol 161:2633-2643

16. Lamas NS, Fontenele RS, Melo FL, Costa AF, Varsani A, Ribeiro SG (2016) Complete Genome Sequence of a Genomovirus Associated with Common Bean Plant Leaves in Brazil. Genome Announc 4

17. Li P, Wang S, Zhang L, Qiu D, Zhou X, Guo L (2020) A tripartite ssDNA mycovirus from a plant pathogenic fungus is infectious as cloned DNA and purified virions. Sci Adv 6:eaay9634

18. Male MF, Kami V, Kraberger S, Varsani A (2015) Genome Sequences of Poaceae-Associated Gemycircularviruses from the Pacific Ocean Island of Tonga. Genome Announc 3

19. Marzano SL, Domier LL (2016) Novel mycoviruses discovered from metatranscriptomics survey of soybean phyllosphere phytobiomes. Virus Res 213:332-342

20. Muhire BM, Varsani A, Martin DP (2014) SDT: a virus classification tool based on pairwise sequence alignment and identity calculation. Plos One 9:e108277

21. Stover BC, Muller KF (2010) TreeGraph 2: combining and visualizing evidence from different phylogenetic analyses. BMC Bioinform 11:7 
22. Varsani A, Krupovic M (2017) Sequence-based taxonomic framework for the classification of uncultured single-stranded DNA viruses of the family Genomoviridae. Virus Evol 3:vew037

23. Wren JD, Roossinck MJ, Nelson RS, Scheets K, Palmer MW, Melcher U (2006) Plant virus biodiversity and ecology. PLoS Biol 4:e80

24. Yu X, Li B, Fu Y, Jiang D, Ghabrial SA, Li G, Peng Y, Xie J, Cheng J, Huang J, Yi X (2010) A geminivirus-related DNA mycovirus that confers hypovirulence to a plant pathogenic fungus. Proc Natl Acad Sci USA 107:8387-8392
25. Zhang W, Olson NH, Baker TS, Faulkner L, Agbandje-McKenna M, Boulton MI, Davies JW, McKenna R (2001) Structure of the maize streak virus geminate particle. Virology 279:471-477

Publisher's Note Springer Nature remains neutral with regard to jurisdictional claims in published maps and institutional affiliations. 